\title{
Low-kV SEM Imaging of Epitaxial Graphene Grown on Various Substrates
}

Iwona Jozwik-Biala $^{1}$, Kacper Gródecki ${ }^{1,2}$, Jacek Baranowski ${ }^{1}$, Włodzimierz Strupinski ${ }^{1}$, Iwona Pasternak $^{1}$, Aleksandra Krajewska ${ }^{1,3}$

${ }^{1}$ Institute of Electronic Materials Technology, Wolczynska 133, 01-919 Warsaw, Poland.

2.Faculty of Physics, University of Warsaw, Hoza 69, 00-681 Warsaw, Poland.

${ }^{3}$ Institute of Optoelectronics, Military University of Technology, Gen. S. Kaliskiego 2, 00-908 Warsaw, Poland.

Graphene is the name given to a planar single sheet of $\mathrm{sp}^{2}$-bonded carbon atoms arranged in a twodimensional (2D) honeycomb lattice. One of methods of obtaining epitaxial graphene is growth on $\mathrm{SiC}$ by sublimation. Epitaxial graphene characterization and device fabrication often require fast and precise identification of the number of graphene layers and their distribution on the substrate.

Graphene single- (SL) and multilayers (ML) grown by sublimation technique on $4 \mathrm{H}$ and $6 \mathrm{H}$ SiC as well as by $\mathrm{CVD}$ on polycrystalline $\mathrm{Cu}$ substrates were subjected to SEM investigation with low energy (less than $1 \mathrm{kV}$ ) electrons using Auriga CrossBeam Workstation (Carl Zeiss) equipped with In-lens SE (true SE1) detector and Energy selective Backscattered electrons (EsB, low-loss BSE) detector, both positioned on the optical axis of the Gemini (TM) column. The main aim of the work was to determine the number of layers grown on the sample using SEM supported by correlative analysis with Raman spectroscopy technique.

It is known that in case the of a stack of graphene ML grown on the Si-terminated $\mathrm{SiC}$, the graphene sheets are stacked in Bernal $\mathrm{AB}$ configuration with respect to each other [1]. That results in an interaction between each of the two sheets forming the delocalized $\pi$-bonding in between, like in a graphite [2]. The contrast in the low-loss BSE of the graphene ML grown on the Si-terminated SiC-4H is not disturbed by the influence of the misorientation of the individual layers. The SEM contrast in this case is only thickness-dependent, so the number of sheets in a ML stack can be easily determined. A correlative imaging of these graphene ML using SEM and Raman spectroscopy was done. The Raman maps showing the FWHM of graphene 2D band are presented in Fig1. The number of graphene layers determined on the basis of the Raman spectroscopy stays in agreement with the results of SEM investigation, e.i. showing one or two graphene layers distributed over the whole observed area.

A comparison of images of graphene ML grown on C-terminated SiC-6H recorded in dual-channel mode using In-Lens (true SE1) and EsB (low-loss BSE) revealed not only topographical contrast, but also information of the number of sheets in a ML stack (based on low-loss BSE contrast). Additionally, an information about the relative orientation/stacking of the graphene ML can be derived from the comparative analysis of the parallel images recorded using both detectors simultaneously. Figure 2 presents the images of graphene ML recorded in SE1 and low-loss BSE contrast simultaneously. The Inlens SE image (left) shows the bright areas, indicating the possibility of higher conductivity of the graphene sheet placed on the top of stack of graphene layers. The same areas appear darker in EsB suggesting random orientation of that sheet over the graphene ML (no Bernal stacking, no interaction and delocalized $\pi$-bonding). 
The EsB detector was also used to perform SEM imaging of graphene layers grown on $\mathrm{Cu}$ foils. That allowed to distinguish easily the characteristic structures of graphene ML growth islands distributed on the $\mathrm{SL}$ covering the $\mathrm{Cu}$ foil. Additional effects, such as the channelling contrast in $\mathrm{Cu}$ grains was observed at low accelarating voltages.

\section{References:}

[1] A Mattausch and O Pankratov, Physical Review Letters 99 (2007) p.076802.

[2] H Jaksch, Proceedings of the Workshop of European Microbeam Analysis Society (2011) p.255.

Figure 1. SEM image (a) and correlative Raman spectroscopy map of 2D band FWHM (b) showing the distribution of the thickness of graphene layers grown on Si-terminated SiC-4H; for FWHM $30-40 \mathrm{~cm}^{-1}$ there is one monolayer graphene, for $40-50 \mathrm{~cm}^{-1}$ one or two, for $50-65 \mathrm{~cm}^{-1}$ two graphene layer and for 65 to $90 \mathrm{~cm}^{-1}$ there are three and more graphene layers.

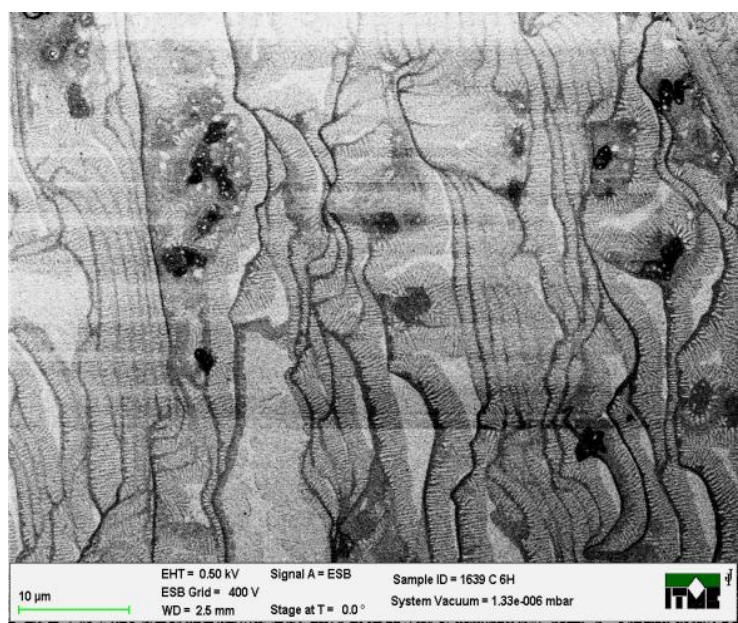

a)

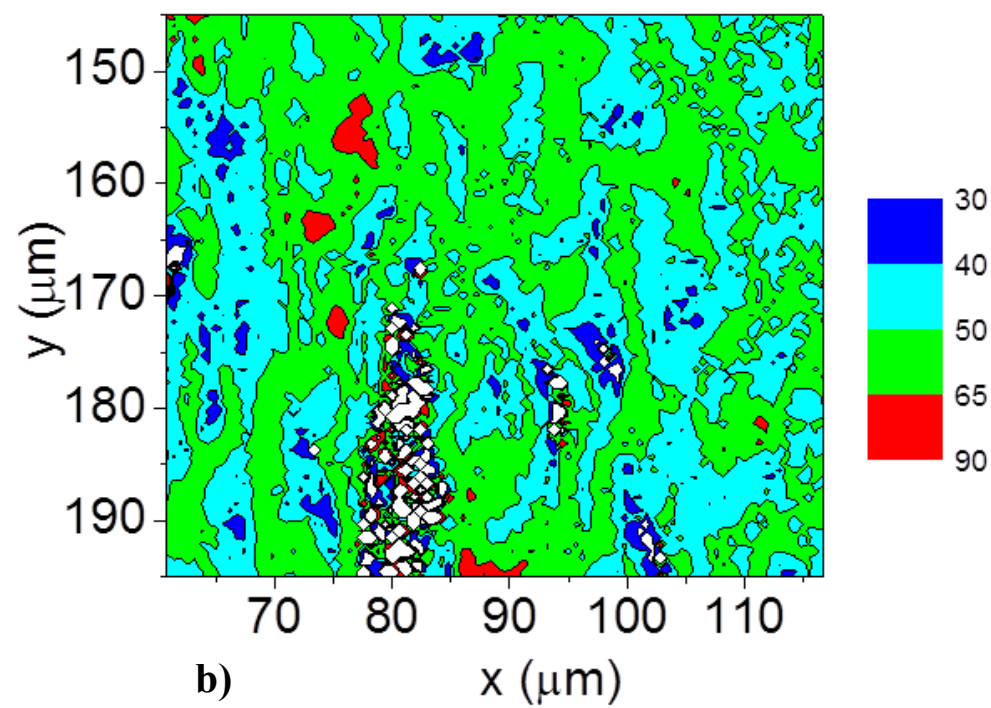

Figure 2. SEM images recorded in dual-channel mode, showing the contrast in the stack of graphene layers grown on $\mathrm{C}$-terminated $\mathrm{SiC}-6 \mathrm{H}$; a) SE1 image, b) low-loss BSE image; areas of random misorientation are brighter in SE1 image and darker in BSE image.

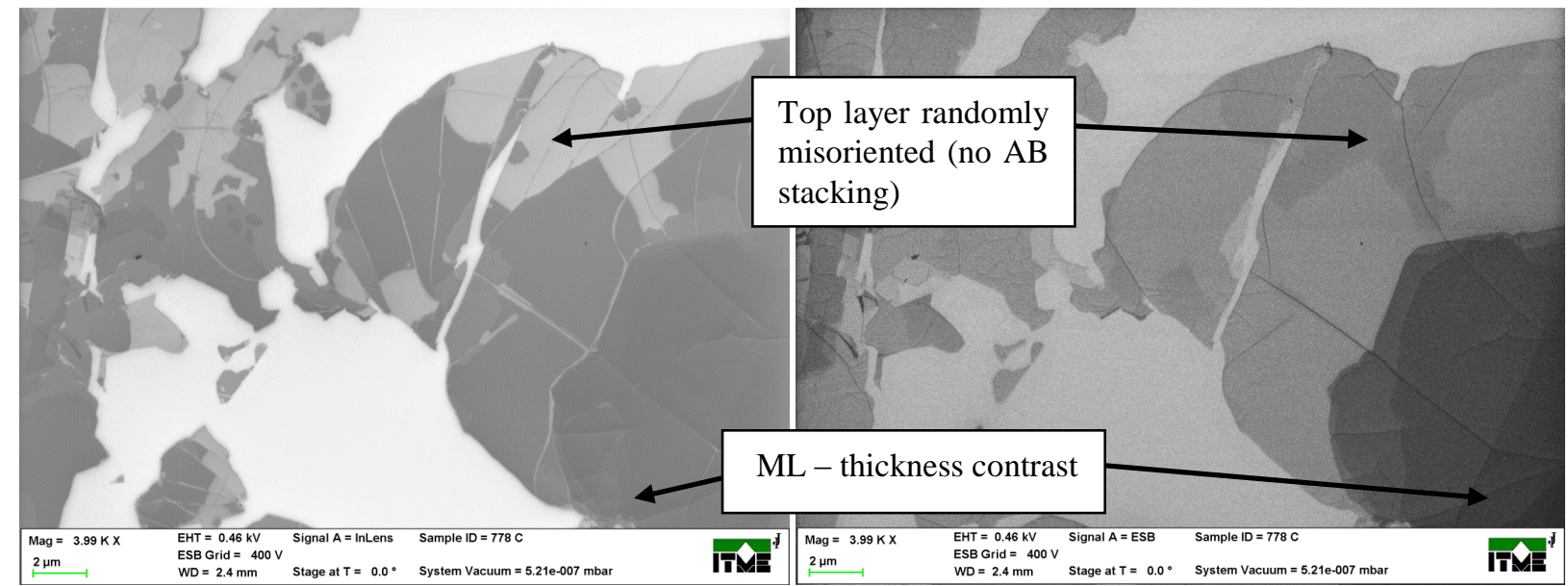

a)

b) 